\title{
The Essential Elements of Successful Innovation
}

\author{
Charles Kalmanek \\ AT\&T Labs Research \\ 180 Park Avenue, Room A147 \\ Florham Park NJ 07932 \\ crk@research.att.com
}

\begin{abstract}
It has become a truism that innovation in the information and communications technology (ICT) fields is occurring faster than ever before. This paper posits that successful innovation requires three essential elements: a need, know-how or knowledge, and favorable economics. The paper examines this proposition by considering three technical areas in which there has been significant innovation in recent years: server virtualization and the cloud, mobile application optimization, and mobile speech services. An understanding of the elements that contribute to successful innovation is valuable to anyone that does either fundamental or applied research in fields of information and communication technology.
\end{abstract}

\section{Categories and Subject Descriptors}

C2.2 [Network Protocols] Protocol architecture; C.4

[Performance of Systems]: Measurement Techniques; D.4.1

[Operating Systems] Process Management; I.2.7 [Artificial

Intelligence] Natural language processing - speech recognition

and synthesis; K.2.1 [Computing Milieux] Computer industry

\section{General Terms}

Design, Economics, Experimentation, Performance.

\section{Keywords}

Application optimization, cloud computing, innovation, speech recognition, virtualization.

\section{INTRODUCTION}

It has become a truism that innovation in the information and communications technology (ICT) fields is occurring faster than ever before. There is ample evidence to support this notion in the pace of change that we see in our everyday lives. In the days of plain old telephone service, Western Electric introduced the rotary dial Model 500 telephone set [9] in 1949. It remained a popular model until the 1980's. In contrast, Apple launched the first iPhone in June 2007. By March 2010 over 100 million iPhones had been sold, and the market share for smartphones in general doubled globally from 3Q 2010 to 3Q 2011 [3]. Another example is that Facebook was founded in 2004. By the end of 2011, it was reported to have over 800 million users. If one looks more deeply at the inventions and technologies that were essential for the success of virtually any innovative ICT product or service, however, the picture that emerges is complex. Many of the inventions essential to today's commercial successes have taken years and even decades before they saw the light of day. Steve Jobs had a vision of having people interact with computers using speech decades ago [13], but it wasn't until 2011 that Siri [10] brought this idea to the mass market. What are the factors that influence how quickly new ideas become widely adopted? What drives successful innovation?

This paper posits that successful innovation requires three essential elements: a need, know-how or knowledge, and favorable economics. If innovation is occurring faster, it's because of a change in one or more of these elements: either there are new needs, we know more, or the economics are better. This article presents several examples illustrating that, in fact, discontinuous changes do occur, leading to an increase in the pace of innovation. In some cases, a break-through invention makes possible what was impossible (new knowledge). In others, the basic knowledge has been around for decades, but changing economics introduce a new need, which leads to a new round of invention and innovation (new need). Even when the knowledge exists, it may take years for technology to evolve to a point where the knowledge can be put to use at a price that people can afford (more favorable economics). Because the economics of the ICT industry is complex, however, some changes take years or even decades before they become widespread.

These elements are neither new nor surprising. The robotics researcher J.F. Engelberger [1] posited an almost identical list of ingredients: 1. A recognized need, 2. Competent people with relevant technology, and 3. Financial support. The present paper addresses the degree to which economic considerations drive successful innovation, though financial support is clearly needed.

An understanding of the factors that contribute to successful innovation is important to anyone that does either fundamental or applied research. If we're doing our jobs as researchers well, we're both confident and fretful creatures. It takes confidence to travel in uncharted waters, but we also have to constantly assess whether we're working on the right problems and whether our work will have impact. The fact that we are researchers means that we are working on problems where the answer to the problem isn't known. In many cases, the problem itself may not even be well defined. To address the unknown, we experiment, we do iterative development, and we root around in the dirt like cave people looking for something shiny. Often, researchers set wildly unachievable goals, work towards those goals for a while, and realize that they have to solve smaller, more tractable problems along the way. The solution to a more tractable problem may ultimately be the thing that has value. The best applied researchers intuitively know how to pick problems where there is a need. Hopefully, they also have an understanding of what solutions are technically feasible and which could be economical on some time horizon. It's then the application of their brainpower which ultimately creates the know-how necessary to move the needle. Increasing our understanding of how successful innovation occurs should make us more effective in our jobs. This is of interest to the entire ACM community, including students, researchers, engineers, and managers.

This paper looks at recent innovation in three technology areas to illustrate how the elements of successful innovation played a role. The three technology areas are server virtualization and the cloud, mobile application optimization, and mobile speech services. 


\section{SERVER VIRTUALIZATION AND THE CLOUD}

The first innovation we'll discuss is server virtualization, part of a trend towards virtualization in enterprise information technology (IT) (e.g., storage virtualization, network virtualization, etc.). It seems clear that virtualization has led to an increase in the pace of innovation in the world of web applications. Software startups these days have virtually zero infrastructure cost on day one they can spin up a bunch of VMs on Amazon EC2 [14] with a credit card and start offering a service. Nonetheless, server virtualization has been around for decades: the first machine to support virtual machines was IBM's System 360, which came to market in the mid 1960's. Other than aggressive marketing, what factors led to the explosion of interest in virtualization in recent years?

We can trace the renewed interest in server virtualization to the mid 2000's. By 2005, web applications were growing dramatically. The typical deployment model was to run each application on a dedicated server, configured with its host operating system. When demand triggered a need for new capacity, the person responsible for the system would buy another server and put it in a rack. X86 based servers were cheap, and IT professionals knew how to rack and stack new servers, so that's what they did. This led to a tremendous growth in the number of x86 based servers. Because demand fluctuates, the average utilization of each dedicated server was typically low. Unfortunately, the economics of cheap servers started to run up against other economic considerations, namely power and space. Servers were becoming more power hungry, demand was driving server deployment, but data centers are engineered for a certain power density. At some point, it's no longer feasible to deploy more servers and you need to build another data center. To illustrate the point, the economics are no longer "can I buy another $\$ 10,000$ server?" they become "can I build another $\$ 80 \mathrm{M}$ data center?" Since virtualization allows multiple applications to share the same physical server, server hardware is used more efficiently. By reducing the number of servers that needed to be deployed, CIOs could avoid massive data center upgrades: they were driven by six and seven figure economic considerations to encourage their staff to use virtualization ${ }^{1}$. This was a strong need, driven by economics.

What about know-how? The fundamentals have been around for decades. By the mid 2005's, server virtualization was being used by companies like VMWare to support development and test environments. The basics were in place. But to fully realize the benefits, IT professionals had to be trained to use virtualization technology to support this new deployment model, and tools were needed to enable developers to use point-and-click provisioning to deploy an application. Addressing the need required investment in software tools and training for IT staff: creation of new knowhow occurred to address the new need. Economics continues to drive even more innovation: sophisticated approaches to resource management such as elastic or cloud computing (which allows developers to quickly scale capacity up and down based on demand) have been combined with pay-as-you-go pricing models.

${ }^{1}$ Power consumption also led to another change, which will not be addressed in detail here. In January 2006 Intel shipped its first Core brand processor. The high power consumption of the earlier NetBurst-based processors, and the resulting inability to increase the clock speed, led to this change to a different architecture.
In fact, Weinman [4] argues that cloud computing is inevitable because of economic considerations. In [5], researchers examine the economic benefits of using cloud computing to reduce the cost of providing disaster recovery and business continuity services.

Where do things stand today? Seven years in, and enterprise IT is still only part way into this journey. Large IT departments have to cope with 1000's of legacy applications written for legacy 32 bit operating systems and older processor generations. Virtualization offers legacy infrastructure consolidation, but migration of legacy applications costs money and the business case has to be sufficiently positive or the migration won't happen. However, the know-how continues to evolve: newer generations of virtualization technology support cloud computing, and multiple service providers are now offering cloud services. As a result, new applications are being written to be deployed in a virtualized environment in which resources can be scaled dynamically. The pace at which the industry moves to this model will continue to be gated by economic considerations, such as training for the developer base and the refresh time for applications, and the maturity of the technology, which relates to know-how.

In summary, the increased interest in virtualization was driven by tremendous growth in web applications in the mid 2000's (need). Virtualization took off due to the economics of large data centers (economics). The fundamental knowledge required to address the need existed, but it was necessary to retrain IT staff and to develop innovative software tools to move the industry in this new direction (know-how). Innovation is occurring rapidly because of the confluence of a recognized need, favorable economics, and the generation of new knowledge. Nonetheless, as a result of both economics and know-how, change will not happen overnight.

\section{MOBILE APPLICATION OPTIMIZATION}

The second innovation is mobile application optimization. Over the past four years, since the emergence of smartphones and widespread deployment of $3 \mathrm{G}$ cellular networks, cellular data traffic has grown by leaps and bounds. New device platforms and new models for monetizing applications have attracted hundreds of thousands of application developers to the mobile Internet. As a measure of the increased demand, AT\&T disclosed that it experienced $8000 \%$ data traffic growth on its wireless network in the three years between 2007 and 2010 [11]. To cope with this growth in demand, AT\&T spent approximately \$28B upgrading its wireless network during the period from 2008 through the first three quarters of 2011 [12]. From the perspective of a network service provider, the high cost of deploying capacity to support this traffic growth created a compelling need to manage traffic growth. Three approaches have emerged to address the need: (1) vendors have developed network-based optimization solutions that promise to reduce network traffic without affecting the user experience; (2) service providers have deployed usage based pricing for cellular data service as a way of incenting users to manage their use of the service; and (3) researchers responded to the need, creating new knowledge that enables application developers and mobile platform providers to develop more efficient applications. This section describes and analyzes the technical and economic considerations of the third approach, application optimization.

In [2], Qian et al. discovered that many mobile application developers were not taking the unique characteristics of the wireless channel into account. Access to the wireless channel is 
controlled by a state machine that introduces a delay in channel access, affecting the user experience. As a result, a timer is used to hold the channel after the application has gone idle, in case the application has more to send. Unfortunately, this wastes both network resources and battery life.

In retrospect, it is obvious that developers would not be aware of the characteristics of the wireless channel. Part of the power of protocol layering is that applications (in principle) don't need to know about lower layer details. Moreover, in the decade and a half since the web and broadband access led to the rapid growth of the Internet, Internet application developers became accustomed to an "always on" channel, and had little incentive to design for efficiency. Despite the exponential growth of Internet traffic, the notion was that Internet service providers would simply deploy more capacity. While this approach has worked (up to a point) in the wired Internet, the cost of keeping up with the explosion in demand for $3 \mathrm{G}$ and $4 \mathrm{G}$ cellular data service are daunting. Cellular data services depend on expensive spectrum licenses, and service is delivered over a shared channel with a capacity that is significantly less than the capacity to my desktop. The difference in the economics of wireless access created a new need.

As mentioned in the introduction, the nature of research is that the solution to a problem, and even the correct problem formulation, may not initially be known. When Qian et al. first discovered how inefficient mobile applications are, they proposed that the radio resource management layer expose an API that would allow applications to relinquish the radio channel when it was no longer needed. Unfortunately, this approach requires changes to both the APIs that the mobile operating system platform exposes, and changes to every application. Due to the complexity of the operating system and application ecosystem, this solution is not likely to happen quickly. Qian et al. theorized that the ideal mobile platform would provide an optimization layer that would sit between applications and the radio resource management layer, and automatically decide on an optimal use of the wireless channel. Application developers would remain blissfully unaware of the wireless channel. While some progress has been made, in its most general form, this problem is difficult and may never be solved. Qian et al. eventually concluded that the solution was to develop a tool that measured what applications were doing, and exposed the results to application developers [6]. In addition, the tool would expose opportunities to piggyback application transactions when the channel was active, rather than setting up the channel for separate transactions, thus conserving both network resources and battery life. The approach was simple and elegant: (1) instrument the mobile platform with a measurement harness (2) run the application through a series of typical use cases (3) upload measurement data to an analysis tool to learn about opportunities to improve application efficiency. The tool is now called Application Resource Optimizer (ARO). During early experiments with several prominent applications, it became apparent that, not only were applications not taking the wireless state machine into consideration, they were also not taking advantage of obvious optimizations, like object caching. These experiments also led to a set of "best practices" that could be provided to application developers.

As described above, the need to reduce traffic is a problem for network service providers. The existence of the ARO tool does not imply that application developers or mobile platform providers will use it. Again, it is important to understand economics and economic incentives. Network service providers can simplistically be said to have two goals: to provide good service to their customers, while controlling costs by optimizing network traffic. The user of a mobile application can be said to have two goals: responsive applications and long battery life. Application developers can be said to have one primary goal, a responsive application, and a secondary goal, long battery life, since this is important to their users. Fortunately, it turns out that the incentives for all three parties are aligned: optimized applications use the network efficiently, conserve battery life, and are more responsive. Without the incentives being aligned, the innovation would not necessarily be successful.

Clearly, the economics of wireless networks stimulated Qian et al. to study mobile applications and create the know-how in the form of the ARO tool and associated best practices. Economics also guided the choice of an end-to-end solution based on application optimization, rather than a network-based solution requiring significant capital expenditures to deploy network equipment. Economic incentives will encourage the use of the tool and the adoption of the best practices. What about training of the application developer community? Fortunately, there is a straightforward distribution path. Providing a tool and appropriate training material fits well with the developer ecosystem model created by the mobile service provider and platform vendors. The ARO tool and best practices were released at the AT\&T Developer Conference in January 2012. In addition to the ARO tool, smartphone vendors are also beginning to provide better tools themselves for consumers to understand the amount of wireless traffic generated by specific mobile applications. It is easy to imagine users avoiding inefficient applications if information about application efficiency is available to them. As with virtualization, mobile application optimization was driven by a recognized need, it required the creation of new knowledge, and it is enabled by favorable economics. The ARO tool and similar capabilities will undoubtedly evolve for years. Similar to virtualization, economic considerations suggest that the widespread adoption of mobile application optimization will take time.

\section{MOBILE SPEECH SERVICES}

The third innovation to be addressed is mobile speech services, such as speech-enabled (voice) search and speech-enabled mobile virtual assistants. While smartphones are rapidly becoming the preferred platform for accessing Internet services for many users, the user experience is limited due to the small form factor of these devices. For many applications like search or messaging, it is far easier for the user to enter information using speech than by typing. For example, AT\&T's YPMobile local business search application lets users use natural language to say a local business name or category. Other applications provide a speech interface to services like SMS and web search. Regulatory considerations are also a factor because of the importance of hands-free, eyesfree operation when users are driving. These considerations have created a new need for large-vocabulary speech recognition that did not exist before.

Before looking in detail at these services, it is useful to take a broader view of the need addressed by large-scale speech recognition and natural language understanding. At the highest level, the need addressed can be expressed as a grand challenge: an automated system that understands human speech and responds appropriately. Obviously, a grand challenge of this nature can occupy computer scientists for decades, and has. Research on speech recognition goes back until at least the 1950's, with experiments at Bell Labs demonstrating recognition of isolated digits for a single speaker. There have been many technical advances in speech recognition over the years, including 
frequency domain analysis in the 1960's, work on speaker independence in the 1970's, the use of Hidden-Markov Models in the 1980's to generate words or phonemes representing the most likely outcome from a statistical model of the "hidden" speech signal, the use of finite state search networks for large-vocabulary applications in the early 1990's, and the use of n-grams to model sequences of words, also in the 1990's [7][8]. These inventions represent only a tiny fraction of the work that has been done.

Rather than looking only at research results, the characteristics of deployed systems are the most relevant measure of state-of-theart, since deployed systems represent innovations that have been developed far enough to economically address a practical need. The emergence of Interactive Voice Response (IVR) systems in the 1970's represented the first large-scale commercial application of speech recognition technology. IVR systems yielded significant reductions in customer care costs. In 1992, AT\&T deployed VRCP, Voice Response Call Processing, which saved AT\&T on the order of \$3B over ten years. In 2000, AT\&T deployed "How May I Help You?," a natural language application that allowed callers to speak naturally rather than selecting from a small set of menu options. This application improved customer satisfaction and reduced customer turnover or "churn" by $18 \%$ following its deployment. In 1999, AT\&T, Lucent, Motorola and IBM formed the Voice XML Forum to create a markup based language for interactive voice response systems and other applications. This was the genesis of the Voice XML standards standardized by the $\mathrm{W} 3 \mathrm{C}$ and used to program IVR systems across the industry today.

We described earlier how the widespread adoption of smartphones that began in the mid 2000's created a new need for speech recognition and language understanding. Small and large companies are beginning to offer mobile speech services that improve the user experience and allow people to easily get access to information and services anywhere and anytime. These services are significantly more complex than traditional IVR services in both scope of the services and the size of the language models being used. The growing adoption of mobile speech services is only possible because of advances in know-how as well as favorable economics.

In terms of know-how, there have been significant advances in acoustic modeling, personalization, adaptation, and language understanding that have occurred over the past decade. In addition, there are three other factors that have made applications like Siri possible today, when they would not have been possible even a few years ago - all of them are associated with economics. The three factors are improvements in memory and processor technology, the widespread deployment of $3 \mathrm{G}$ wireless networks, and the emergence of new models for monetizing mobile applications.

The first of these factors is due to Moore's Law. Largevocabulary speech recognition systems use statistical models representing the space of potential utterances that can be recognized. To train large models efficiently requires a large amount of in-core memory. In 2005, a typical 32-bit x86 server had a memory size that was limited to $4 \mathrm{~GB}$, due to the 32-bit address space that was available in processors at that time. In 2011, a 64-bit multi-core Xeon processor can support in-core memory in the 100's of Gigabytes. This vastly larger memory size makes it possible to train the large statistical models necessary for a usable search application at reasonable cost To support real-time recognition, improvements in processor speed are also important. While clock speed is no longer the best metric, it is still useful to note that in 2005, the Pentium Pro supported clock speeds of 150-200 MHz. In 2011, clock speeds of 2-3 GHz were common. In addition to the new know-how created by recent research advances, Moore's Law made largevocabulary real-time speech recognition economically feasible.

The second factor is the widespread deployment of $3 \mathrm{G}$ cellular data services. While smartphones can support embedded speech recognition, the complexity and number of language models supported is necessarily limited. The most sophisticated applications are built by streaming speech from the device over the wireless channel to a server in a data center, which then does speech recognition, runs application layer code, and returns the results. Since most of these applications are interactive in nature, latency is important, but the latency introduced by the $3 \mathrm{G}$ wireless channel is acceptable. These applications would not have been possible over the $2 \mathrm{G}$ wireless channels available even a few years ago.

The third factor is not unique to mobile speech services, but certainly has helped them along. The mobile web has created new revenue models such as advertising based services, which have fueled innovation; and the app store model has created a frictionless distribution model for application developers. In the case of Siri, the economic driver is the sale of devices that support a novel and powerful user experience.

Mobile speech services illustrate the principle yet again: a new need, combined with new know-how and a change in the underlying economics, have enabled an entirely new class of large-vocabulary speech recognition application.

\section{OTHER CONSIDERATIONS}

The preceding sections provided a detailed look at the three essential elements of successful innovation. There are several important additional considerations, with global ramifications, that have influenced all of the technology areas that we have discussed. Historically, the timescale of innovation in the communications part of the ICT industry was heavily influenced by a lengthy standardization process. Inter-operability of network equipment clearly requires standardization, but the emergence of the global Internet and web protocols have enabled developers to innovate rapidly at the edge of the network and achieve global reach, without waiting for standards to emerge. The ability to instantly deploy applications in the cloud and scale capacity on demand will accelerate this trend. In addition to the Internet and the cloud, the app store model clearly speeds innovation by making it easier for application developers to monetize applications. Finally, the presence of a vibrant open source community has both accelerated knowledge creation through collaborative development, and significantly lowered the cost of developing software compared with the licensing model that dominated software development until the late 1990's.

\section{CONCLUSION}

We've considered three innovations that have gotten a great deal of attention in the last few years: server virtualization and the cloud, mobile application optimization, and mobile speech services. In each of these areas, a need, knowledge and favorable economics contributed to successful innovation. In the case of virtualization, the economics of power and space sparked a significant change in the way that applications are deployed. This change leveraged technology that had been around for decades, but it also stimulated creation of new knowledge and innovation. While server virtualization and the cloud have received significant attention, enterprise IT practices will not change overnight. The 
pace of change will be gated by the lifecycle of server and application upgrades, and by the need to invest in retraining IT staff. In the case of mobile application optimization, there has been tremendous growth in mobile applications in a very short time. Inefficient applications and the cost of mobile networks created a need, which stimulated the creation of new knowledge. The economic incentives look good for more efficient applications to emerge, but the pace of this change will be driven by the extent to which these economic incentives hold true, and by the lifecycle of retraining the developer community. Finally, mobile devices created a need for an improved user interface based on speech recognition. This need could be satisfied due to significant advances in knowledge, and favorable economics due to Moore's Law, 3G wireless networks, and new models for monetizing applications. As a result, large-scale speech recognition systems have crossed a threshold where applications that would not have been economical even five years ago can now be developed.

An understanding of the elements that contribute to successful innovation is important to anyone that does either fundamental or applied research in the ICT fields. The best research is relevant: it addresses a need. To give some examples, the need could be motivated by a fundamental scientific problem; an engineering problem such as memory constraints or system performance bottlenecks; an operational problem such as configuration or management; or by economic considerations, as with server virtualization and mobile application optimization. It can also be motivated by a human need, which contributed to the emergence of mobile speech services. In an industrial research environment, one can often get good ideas about needs simply by talking to one's colleagues in marketing, development or operations. Sometimes the most meaningful research question requires reframing, abstracting, or generalizing the problem from the way that it is originally presented. In addition to motivating problems to work on, economics also has a huge impact on whether and when a technical idea will become a successful innovation. This suggests that researchers should think about the economic factors at work in their field of study, since an understanding of the underlying economics is a crucial consideration in whether their work will be adopted and used - the hallmark of successful innovation.

\section{ACKNOWLEDGMENTS}

The author gratefully acknowledges Simon Crosby, Mazin Gilbert, Krish Prabhu, Juergen Schroeter, Shubho Sen, Oliver Spatscheck, and Jay Wilpon for conversations that contributed to the material in this article.

\section{REFERENCES}

[1] Engelberger, J. F., 1982. Robotics in practice: Future capabilities, Electronic Servicing \& Technology magazine.

[2] Qian, F; Wang, Z.; Gerber, A.; Mao, M.; Sen, S.; Spatscheck, O.; 2010. TOP: Tail Optimization Protocol for Cellular Radio Resource Optimization, $18^{\text {th }}$ IEEE International Conference on Network Protocols, Kyoto.

[3] Strategy Analytics report on Wireless Operator Strategies; December 12, 2011.

[4] Weinman, J., 2011. Mathematical Proof of the Inevitability of Cloud Computing, http://joeweinman.com/Resources/Joe Weinman Inevitabilit y_Of_Cloud.pdf

[5] Wood, T.; Cecchet, E.; Ramakrishnan, K.K.; Shenoy, P.; van der Merwe, J., Venkataramani, A., 2010. Disaster Recovery as a Cloud Service: Economic Benefits and Deployment Challenges; $2^{\text {nd }}$ USENIX Workshop on Hot Topics in Cloud Computing. Boston, MA.

[6] Qian, F.; Wang, Z.; Gerber, A.; Mao, M.; Sen, S.; Spatscheck, O.; 2011. Profiling Resource Usage for Mobile Applications: A Cross-Layer Approach, ACM Mobisys, Washington, DC.

[7] Rabiner, L., 1989. A Tutorial on Hidden-Markov Models and Selected Applications in Speech Recognition. Proceedings of the IEEE. 257-286.

[8] Jelinek, F.; Neufeld, E.; Statistical Methods in Speech Recognition, McGraw-Hill. Cambridge, Mass.: MIT Press. 283p. ISBN 0-262-10066-5.

[9] Model 500 telephone, http://en.wikipedia.org/wiki/Model_500_telephone

[10] Siri, http://www.apple.com/iphone/features/siri.html

[11] Mobile traffic growth, http://www.cultofmac.com/91703/att8000-mobile-data-growth-because-of-iphone-and-ipad-iswhy-were-buying-t-mobile/

[12] Internal communication.

[13] Isaacson, W., Steve Jobs, Simon and Schuster, 2011, ISBN13: 978-1451648539

[14] Amazon Elastic Compute Cloud, http://aws.amazon.com/ec2/ 